\title{
Effect of ATM on inflammatory response and autophagy in renal tubular epithelial cells in LPS-induced septic AKI
}

\author{
CHENFEI ZHENG ${ }^{1 *}$, YING ZHOU ${ }^{1 *}$, YUEYUE HUANG ${ }^{2}$, BICHENG CHEN $^{3}$, MINMIN WU $^{3}$,

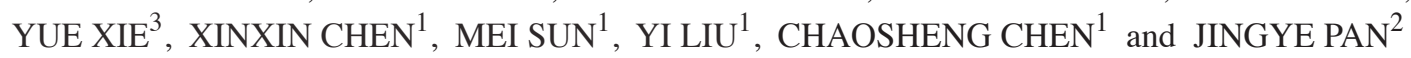 \\ Departments of ${ }^{1}$ Nephrology and ${ }^{2}$ Intensive Care Unit, The First Affiliated Hospital of Wenzhou Medical University; \\ ${ }^{3}$ Zhejiang Provincial Top Key Discipline in Surgery, Wenzhou Key Laboratory of Surgery, Department of Surgery, \\ The First Affiliated Hospital of Wenzhou Medical University, Wenzhou, Zhejiang 325000, P.R. China
}

Received September 13, 2018; Accepted July 29, 2019

DOI: $10.3892 /$ etm.2019.8115

\begin{abstract}
The aim of the present study was to explore the role of ataxia-telangiectasia mutated (ATM) in lipopolysaccharide (LPS)-induced in vitro model of septic acute kidney injury (AKI) and the association between ATM, tubular epithelial inflammatory response and autophagy. The renal tubular epithelial cell HK-2 cell line was cultured and passaged, with HK-2 cell injury induced by LPS. The effects of LPS on HK-2 cell morphology, viability, ATM expression and inflammation were observed. Lentiviral vectors encoding ATM shRNA were constructed to knock down ATM expression in HK-2 cells. The efficiency of ATM knockdown in HK-2 cells was detected by western blot analysis and reverse transcription-quantitative PCR (RT-qPCR). HK-2 cells transfected with the ATM shRNA lentivirus were used for subsequent experiments. Following ATM knockdown, corresponding controls were set up, and the effects of ATM on inflammation and autophagy were detected in HK-2 cells using RT-qPCR, western blotting and ELISA. After LPS stimulation, the HK-2 cells were rounded into a slender or fusiform shape with poorly defined outlines. LPS treatment reduced cell viability in a partly dose-dependent manner. LPS increased the expression of tumor necrosis factor- $\alpha$, interleukin (IL)- $1 \beta$ and IL-6, with the levels reaching its highest value at $10 \mu \mathrm{g} / \mathrm{ml}$. IL-6 and IL-1 $\beta$ expression
\end{abstract}

Correspondence to: Dr Jingye Pan, Department of Intensive Care Unit, The First Affiliated Hospital of Wenzhou Medical University, Fanhai West Road, Wenzhou, Zhejiang 325000, P.R. China

E-mail: panjingye@wzhospital.cn

Dr Bicheng Chen, Zhejiang Provincial Top Key Discipline in Surgery, Wenzhou Key Laboratory of Surgery, Department of Surgery, The First Affiliated Hospital of Wenzhou Medical University, Fanhai West Road, Wenzhou, Zhejiang 325000, P.R. China

E-mail: bisonch@163.com

${ }^{*}$ Contributed equally

Key words: septic acute kidney injury, inflammation, ataxia-telangiectasia mutated, lipopolysaccharide, autophagy increased with increasing LPS concentration. These findings suggest that LPS reduced HK-2 cell viability whilst increasing the expression of inflammatory factors. Following transfection with ATM shRNA, expression levels of key autophagy indicators microtubule associated protein 1 light chain $3 \alpha$ I/II ratio and beclin-1 in the two ATM shRNA groups were also significantly reduced compared with the NC shRNA group. In summary, downregulation of ATM expression in HK-2 cells reduced LPS-induced inflammation and autophagy in sepsis-induced AKI in vitro, suggesting that LPS may induce autophagy in HK-2 cells through the ATM pathway leading to the upregulation of inflammatory factors.

\section{Introduction}

Sepsis is a systemic inflammatory response syndrome (SIRS) caused by infection, which is an excessive inflammatory response as a result of the uncontrolled release of inflammatory mediators (1). In SIRS and secondary tissue damage, cytokines are released into the circulation in a dysregulated manner, causing hemodynamic instability, extensive tissue damage and fatal multiple organ dysfunction (2). The number of organ failures in patients with sepsis is significantly associated with mortality (3). The kidney is one of the most vulnerable organs in the body to sepsis (4). Acute kidney injury (AKI) is the most common and serious complication of sepsis and it is often used as an independent risk factor for predicting mortality (1). According to research statistics, in the intensive care unit, $\sim 42 \%$ of patients with sepsis are afflicted with different degrees of AKI (5). AKI is a clinical syndrome that results in severe tubular damage with mortality rates ranging between 24 and $62 \%$ (6). In the case of AKI, the mortality rate of sepsis can reach $70 \%$ due to a lack of effective therapy strategies $(7,8)$.

The pathogenesis of septic AKI is closely associated with renal hemodynamic abnormalities, inflammatory injury, apoptosis and adaptive mechanisms. During adaptation, the host reduces its sensitivity to inflammation-induced tissue damage which mainly affects metabolism, resists injury, guides tissue repair and promotes organ recovery by altering cell signaling pathways (9). In particular, the main cause of septic AKI is the lipopolysaccharide (LPS)-mediated apoptosis of renal 
tubular epithelial cells (RTEC) (10). LPS is a component of the outer membrane of Gram-negative bacteria that is involved in the pathogenesis of sepsis-induced AKI $(11,12)$. LPS has the ability to stimulate severe inflammatory reactions, frequently resulting in the release of a large number of inflammatory cytokines, including tumor necrosis factor- $\alpha(\mathrm{TNF}-\alpha)$, interleukin (IL)-1 $\beta$ and IL-6. The subsequent inflammatory reaction can in turn lead to oxidative stress, mitochondrial damage and energy depletion, and eventually the apoptosis of RTEC. It has been previously reported that kidney injury in AKI animal models can be markedly ameliorated by reducing the intensity of inflammatory reactions; therefore, effective removal of the inflammatory mediators, including TNF- $\alpha$, IL-15 and IL-6, as an effective means of treating septic AKI $(13,14)$.

RTEC death in septic AKI is manifested as necrosis, apoptosis and autophagy. Cell necrosis is a cell lysis process typically due to pathology or trauma, apoptosis is a cell death mechanism occurring in an orderly, controlled manner, while autophagy is a metabolic process of cell self-defense that is distinct from apoptosis, and is important for the turnover of intracellular substances in eukaryotes (15). Autophagy is a process in which cells form autophagic lysosomes to degrade their own damaged organelles such as mitochondria, and other macromolecules. Autophagy can satisfy cellular metabolic requirements and the renewal of organelles, and is an important regulatory mechanism for cell growth, differentiation and death (16). Regulation of the balance of pro- and anti-inflammatory factors serves a pivotal role in the severity of the inflammatory response. Studies have shown that autophagy can antagonize apoptosis and protect RTECs from LPS-mediated damage, and the inhibition of autophagy can aggravate LPS-mediated AKI $(17,18)$.

Ataxia-telangiectasia mutated (ATM) belongs to the phosphatidyl inositol-3-kinase-like kinase family of proteins in mammalian cells, which also includes ataxia telangiectasia and rad3 related, DNA-dependent protein kinase and mTOR. Mutated or inactivated forms of ATM have been identified in ataxia telangiectasia patients (19). ATM is one of the key transducers of the DNA double-stranded break response and serves critical roles in early signal transduction through cell cycle checkpoints. Homologs of ATM are present in all eukaryotic cell types examined to date, including budding and fission yeasts (20). A previous study suggested that persistent inflammation leads to DNA damage in RTEC and further activation of ATM $(21,22)$. In addition, another study in ischemia reperfusion kidney injury models reported that AMP-activated protein kinase (AMPK) activated the ATM-AMPK-tuberous sclerosis complex 2 (TSC2)-mTOR pathway, triggering autophagy (23). However, it remains unclear whether the inflammatory reaction in septic AKI leads to increased ATM expression or increased autophagy.

In the present study, changes in ATM expression and levels of cell autophagy in an in vitro RTEC model of septic AKI was assessed using lentiviral transfection to knock down ATM expression in HK-2 cells. The results of immunofluorescence and western blotting suggest that in septic AKI, ATM expression is elevated, which increases autophagy in RTEC. In addition, downregulation of ATM expression in HK-2 cells reduced the expression levels of inflammatory factors and autophagy in LPS-induced septic AKI cells. The aim of the current study was to investigate the mechanism by which the inflammatory response of septic AKI mediates RTEC damage, thus providing a new strategy for the therapeutic intervention of septic AKI.

\section{Materials and methods}

Cell lines. The human RTEC line HK-2 was obtained from Cell Culture Center of the Basic Institute of Medical Sciences, Peking Union Medical College.

Cell culture and passage. The HK-2 cell line was cultured in DMEM (Gibco; Thermo Fisher Scientific, Inc.) containing $10 \%$ fetal bovine serum (FBS; Biochrom, Ltd.) and incubated at $37^{\circ} \mathrm{C}$ in a humidified atmosphere with $5 \% \mathrm{CO}_{2}$. The cells were sub-cultured at $80 \%$ confluence, which were removed from the incubator and the original medium in the dish was discarded. Cells were rinsed using $3 \mathrm{ml}$ PBS and digested by treatment with $1 \mathrm{ml}$ trypsin for $1-2 \mathrm{~min}$ at $37^{\circ} \mathrm{C}$. Digestion was terminated using $2 \mathrm{ml}$ DMEM medium, and the cell suspension was subsequently centrifuged at $450 \mathrm{x}$ g for $5 \mathrm{~min}$ at $4^{\circ} \mathrm{C}$. Supernatant was discarded and cells were resuspended in $2 \mathrm{ml}$ corresponding DMEM medium to obtain a single cell suspension. Cells were seeded into different dishes/microplates at different densities for subsequent experimentation, as described below.

Induction of HK-2 cell injury using LPS. HK-2 cells were cultured under the above conditions. At $100 \%$ confluency, cells were digested and centrifuged at $480 \mathrm{x}$ g for $8 \mathrm{~min}$ at room temperature. The supernatant was subsequently discarded and the cells were resuspended in $10 \mathrm{ml}$ PBS, counted and seeded into six-well plates. At $80 \%$ confluency the cells were washed three times in PBS and then cultured in DMEM/F12 (Gibco; Thermo Fisher Scientific, Inc.) medium without FBS for $6 \mathrm{~h}$. LPS (Sigma-Aldrich; Merck KGaA) diluted in DMEM/F12 without antibiotics was then added to the cells at final concentrations of $1,10,20$ and $30 \mu \mathrm{g} / \mathrm{ml}$ followed by further incubation for $0,6,12,24 \mathrm{~h}$. Finally, the optimal concentration $(10 \mu \mathrm{g} / \mathrm{ml})$ and the optimal stimulation time $(24 \mathrm{~h})$ were selected for subsequent experiments. In the control group PBS was added instead of LPS.

Cell proliferation assay. Cell proliferation was analyszd using the Cell Counting Kit-8 (CCK-8; Dojindo Molecular Laboratories, Inc.). The HK-2 cells in the logarithmic growth phase were seeded into a 96-well plate at a density of $2 \times 10^{4}$ cells/well. Following incubation with different concentrations of LPS solution $(1,10,20$ and $30 \mu \mathrm{g} / \mathrm{ml})$ and whole medium (DMEM + 10\% fetal bovine serum), HK-2 cells were continuously stimulated. The control group was treated with an equal volume of PBS, whereas the blank control consisted of medium only with no cells. After the completion of LPS treatment, CCK-8 solution $(10 \mu \mathrm{l})$ was added into each well and the mixture was incubated for $2 \mathrm{~h}$. Absorbance values (OD value) at $450 \mathrm{~nm}$ was measured in each well using an enzyme-labeled instrument. The results were obtained from three independent experiments in triplicate. The OD value is considered to be directly proportional to the number of viable cells contained in the culture system. 
Table I. RT reaction solution.

\begin{tabular}{lc}
\hline Reagent & Amount added per reaction \\
\hline $5 X$ RT buffer & $4 \mu 1$ \\
$10 \mathrm{mM}$ dNTPs & $2 \mu 1$ \\
RNasin & $0.5 \mu 1$ \\
M-MLV-RT & $1 \mu 1$ \\
DEPC $\mathrm{H}_{2} \mathrm{O}$ & $3.5 \mu \mathrm{l}$ \\
\hline
\end{tabular}

RT, reverse transcription; dNTP, deoxynucleotide triphosphosphate; M-MLV-RT, Molony-urine leukemia virus reverse transcriptase; DEPC, diethyl pyrocarbonate.

Inflammatory factor detection by reverse transcription-quantitative PCR (RT-qPCR). HK-2 cells in good condition were first collected and cultured, and the levels of ATM, TNF- $\alpha$, IL-1 $\beta$ and IL- 6 mRNA expression in HK-2 cells were detected using RT-qPCR. Following $24 \mathrm{~h}$ of LPS stimulation, total RNA was extracted using TRIzol reagent according to the manufacturer's protocol (Invitrogen; Thermo Fisher Scientific, Inc.). The concentration of the extracted RNA was measured using an ultraviolet analyzer. Reverse transcription of RNA into cDNA was performed using M-MLV reverse transcriptase (Promega Corporation) in a reaction mix prepared according to manufacturer's protocol (Table I), and the resultant cDNA was stored at $-80^{\circ} \mathrm{C}$. The setup of a qPCR reaction system (SYBR ${ }^{\circledR}$ Premix Ex Taq ${ }^{\mathrm{TM}}$; Takara Biotechnology Co., Ltd.) is provided in Table II. The sequences of the primer pairs used for RT-qPCR were as follows: ATM forward, 5'-ATAGATTGT GTAGGTTCCGATGG-3' and reverse, 5'-CATCTTGTCTCA GGTCATCACG-3'; TNF- $\alpha$ forward, 5'-ACCTCTCTCTAA TCAGCCCTCT-3' and reverse, 5'-GGGTTTGCTACAACA TGGGCTA-3'; IL-1 $\beta$ forward, 5'-GCAATGAGGATGACT TGTTCTTTG-3' and reverse, 5'-CAGAGGTCCAGGTCC TGGAA-3'; IL-6 forward, 5'-AGCCACTCACCTCTTCAG AAC-3' and reverse, 5'-ACATGTCTCCTTTCTCAGGGC-3', $\beta$-actin (reference) forward, 5'-CCTGACTGACTACCTCAT GAAG-3' and reverse, 5'-GACGTAGCACAGCTTCTCCTT A-3'. The following thermocycling conditions were used for qPCR: Initial denaturation at $95^{\circ} \mathrm{C}$ for $15 \mathrm{sec} ; 45$ cycles of $95^{\circ} \mathrm{C}$ for $5 \mathrm{sec}$ and $60^{\circ} \mathrm{C}$ for $30 \mathrm{sec}$. The absorbance values of the fluorophores were read each time during the extension phase. A melting curve was then prepared and an initial denaturation of the template DNA at $95^{\circ} \mathrm{C}$ for $1 \mathrm{~min}$ after the end of PCR. It was then cooled to $55^{\circ} \mathrm{C}$ to allow sufficient binding to the DNA duplex. From $55^{\circ} \mathrm{C}$ to $95^{\circ} \mathrm{C}$, each step was increased by $0.5^{\circ} \mathrm{C}$ for $4 \mathrm{sec}$ while the absorbance was being read. The $2^{-\Delta \Delta \mathrm{Cq}}$ method (24) was used to calculate the relative expression of ATM, TNF- $\alpha$, IL- $1 \beta$ and IL- 6 mRNA against $\beta$-actin. All samples were tested in triplicate.

ELISA. Changes in expression levels of inflammatory factors, including IL-1 $\beta$, IL-6 and TNF- $\alpha$, in response to LPS were detected using ELISA kits (cat. nos. 558279, 555220 and 555212, respectively; BD Pharmingen; BD Biosciences). Briefly, $50 \mu \mathrm{l}$ of the capture monoclonal antibody (mAb) was applied to the ELISA plate, and the plate was incubated at
Table II. Quantitative PCR reaction system.

\begin{tabular}{lc}
\hline Reagent & Amount added per tube \\
\hline SYBR premix ex taq & $10 \mu 1$ \\
ROX Reverse Dye $(50 \mathrm{X})$ & $0.4 \mu \mathrm{l}$ \\
Forard primer $(2.5 \mu \mathrm{M})$ & $0.5 \mu 1$ \\
Reverse primer $(2.5 \mu \mathrm{M})$ & $0.5 \mu 1$ \\
cDNA & $1.0 \mu 1$ \\
$\mathrm{H}_{2} \mathrm{O}$ & $7.6 \mu 1$ \\
\hline
\end{tabular}

$37^{\circ} \mathrm{C}$ for $1 \mathrm{~h}$ and washed three times with PBS supplemented with Tween-20 (PBS-T). The plate was then treated with $50 \mu 1$ bovine serum albumin (BSA; $10 \mathrm{mg} / \mathrm{ml}$; Proliant, Inc.) and $50 \mu \mathrm{l} \mathrm{FBS}$, followed by incubation at $37^{\circ} \mathrm{C}$ for $1 \mathrm{~h}$. A total of $50 \mu \mathrm{l}$ biotin-conjugated detector $\mathrm{mAb}$ was added to each well and incubated at $37^{\circ} \mathrm{C}$ for $1 \mathrm{~h}$, followed by probing using an avidin-horseradish peroxidase (HRP) solution $(10 \mathrm{mg} / \mathrm{ml})$. After final rinsing with PBS-T, $50 \mu$ l tetramethylbenzidine substrate solution $(10 \mathrm{mg} / \mathrm{ml})$ was added to start the color reaction of the antigen-antibody complex, which was then stopped by adding $50 \mu \mathrm{l}_{2} \mathrm{SO}_{4}(10 \mu \mathrm{mol} / \mathrm{ml})$. Final absorbance at a wavelength of $450 \mathrm{~nm}$ was measured using an automated ELISA reader (Bio-Rad Laboratories, Inc.). The concentration of each cytokine was determined by comparing the optical densities at $450 \mathrm{~nm}$ to a standard curve. To further confirm the role of autophagy in HK-2 cell injury induced by LPS, HK-2 cells were pretreated with $10 \mathrm{~mol} / 1$ 3-methyladenine (3-MA; Sigma-Aldrich; Merck KGaA) at room temperature, followed by treatment with $10 \mu \mathrm{g} / \mathrm{ml}$ LPS for $30 \mathrm{~min}$.

Construction of lentivirus-mediated ATM-knockdown system. The mRNA sequence of ATM was obtained from the NCBI database (https://www.ncbi.nlm.nih.gov/gene/472) and four shRNA sequences were designed according to Table III, and the constructs were named accordingly: PLVE2142, PLVE2143, PLVE2144 and PLVE2145. The double-strand DNA oligonucleotide containing the interference sequence was synthesized by Shanghai GeneChem Co., Ltd. and cloned into the pLV-GFP lentiviral vector (Hanheng Biotechnology Shanghai Co., Ltd.) using EcoRI-HF and AgeI-HF restriction sites. The vectors were prepared using a Plasmid Minipreparation kit (cat. no. KL060; Shanghai Kang Lang Biological Technology Co., Ltd.) and detected using $2 \%$ agarose gel electrophoresis with ethidium bromide staining.

The experiments were performed in five groups: Negative control (NC), PLVE2142, PLVE2143, PLVE2144 and PLVE2145 groups. Using the Lipofectamine ${ }^{\circledR} 3000$ reagent (Invitrogen; Thermo Fisher Scientific, Inc.) according to manufacturer's protocol, DNA-liposome complexes were prepared at $4^{\circ} \mathrm{C}$ to a final volume of $1 \mu \mathrm{g} / \mu \mathrm{l}$ and added to HK-2 cells $(1 \mu \mathrm{g} / \mathrm{ml})$, transfection was performed for $6 \mathrm{~h}$ at room temperature. Further experiments were performed $48 \mathrm{~h}$ post-transfection.

Cellular infection with recombinant lentiviruses expressing ATM shRNA. Cells were seeded into six-well plates at a density of $1 \times 10^{5}$ cells/well. Once cells reached $30 \%$ confluence, the 
Table III. Sequences of ATM shRNAs.

Primer

Sequence $\left(5^{\prime}-3^{\prime}\right)$

\section{SH-NC-F}

SH-ATM-R

SH-ATM-F1

SH-ATM-R1

SH-ATM-F2

SH-ATM-R2

SH-ATM-F3

SH-ATM-R3

SH-ATM-F4

SH-ATM-R4
CCGGGAGGTCAAACCTAGAAAGCTCCTCGAGGAGCTTTCTAGGTTTGACCTCTTTTTTG AATTCAAAAAAGAGGTCAAACCTAGAAAGCTCCTCGAGGAGCTTTCTAGGTTTGACCTC CCGGGAGGTCAAACCTAGAAAGCTCCTCGAGGAGCTTTCTAGGTTTGACCTCTTTTTTG AATTCAAAAAAGAGGTCAAACCTAGAAAGCTCCTCGAGGAGCTTTCTAGGTTTGACCTC CCGGGCTGCAGAGTCAATCAATAGACTCGAGTCTATTGATTGACTCTGCAGCTTTTTTG AATTCAAAAAAGCTGCAGAGTCAATCAATAGACTCGAGTCTATTGATTGACTCTGCAGC CCGGGAGCTCTTCAGGTCTAAATCACTCGAGTGATTTAGACCTGAAGAGCTCTTTTTTG AATTCAAAAAAGAGCTCTTCAGGTCTAAATCACTCGAGTGATTTAGACCTGAAGAGCTC CCGGGTCATATAGGAAGTAGAGGAACTCGAGTTCCTCTACTTCCTATATGACTTTTTTG AATTCAAAAAAGTCATATAGGAAGTAGAGGAACTCGAGTTCCTCTACTTCCTATATGAC

ATM, ataxia telangiectasia mutated; F, forward; R, reverse; SH or shRNA, short harpin RNA.

recombinant lentiviruses PLVE2142, PLVE2143, PLVE2144 or PLVE2145 were used to transfect HK-2 cells $(8 \mu \mathrm{g} / \mathrm{ml}$ Polybrene; Sigma-Aldrich; Merck KGaA). The cells were then transferred to DMEM containing $2 \%$ horse serum (Gibco; Thermo Fisher Scientific, Inc.), and the lentivirus:polybrene mixture was added to cells. The cells were subsequently incubated in $37^{\circ} \mathrm{C}$ for $6 \mathrm{~h}$ before the media was replaced with media free of lentivirus. Following $48 \mathrm{~h}$ of further culture at $37^{\circ} \mathrm{C}$, images of the cells were taken using a fluorescence microscope. Western blot analysis and RT-qPCR were used to determine the efficiency of ATM knockdown HK-2 cells, and the appropriate multiplicity of infection of ATM lentiviral interference vector was selected for subsequent experiments after incubation of $24 \mathrm{~h}$. After the ATM gene was silenced by shRNA (MOI, 10), HK-2 cells were stimulated with $10 \mu \mathrm{g} / \mathrm{ml}$ LPS for $24 \mathrm{~h}$ at $4^{\circ} \mathrm{C}$, following which the effects of ATM knockdown on the mRNA expression of cellular inflammatory factors and autophagy was examined.

Immunofluorescence staining. HK-2 cells were transfected with pDSRed-LC3 the lentivirus (MOI, 20) for $48 \mathrm{~h}$ and then fixed with $4 \%$ paraformaldehyde (Sigma-Aldrich; Merck $\mathrm{KGaA}$ ) for $15 \mathrm{~min}$ at room temperature, washed three times with PBS for a total of 10 min each and permeabilized with pre-chilled $\left(-20^{\circ} \mathrm{C}\right) 70 \%$ ethanol for $20 \mathrm{~min}$. The cells were then blocked with $8 \%$ BSA diluted in PBS for $1 \mathrm{~h}$ and washed with PBS. Following incubation for $2 \mathrm{~h}$, transfected HK-2 cells were labeled with DAPI $(5 \mathrm{mg} / \mathrm{l})$ and incubated with pDSRed-LC3 (cat. no. 38171200626; purchased from REBIO; Shanghai Shengwu Gongcheng Co., Ltd.) overnight at $4^{\circ} \mathrm{C}$. Subsequently, the treated cells were washed three times with PBS and incubated at room temperature with AlexaFluor ${ }^{\circledR}$ 488-conjugated anti-rabbit secondary antibody (dilution 1:1,000; cat. no. A-11008; Thermo Fisher Scientific, Inc) for $1 \mathrm{~h}$. The cells attached to glass slides were removed from the 24-well plates in the dark room and placed faced up on a blotting paper, where a drop of antifade mounting medium (Invitrogen; Thermo Fisher Scientific, Inc.) was added. After the cells were dried, they were imaged using a Leica TCS SP8 laser confocal microscope (Leica Microsystems, Inc.).
Western blot analysis of HK-2 cells autophagy. Western blot analysis was performed to measure the expression of beclin-1 and LC3-II/I, proteins associated with autophagy (25). HK-2 cells were lysed using RIPA lysis buffer (Teknova, Inc.) at $4^{\circ} \mathrm{C}$ for $10-15 \mathrm{~min}$. Protein concentration was determined using bicinchoninic acid assay method. Equal amounts of $50 \mu \mathrm{g}$ protein extract were separated by $10 \%$ SDS-PAGE and transferred onto polyvinylidene fluoride membranes. The membranes were blocked with $5 \%$ nonfat milk diluted in Tris-buffered saline containing $0.1 \%$ Tween-20 at room temperature for $90 \mathrm{~min}$. The membranes were then immunoblotted for Beclin1 (1:1,000; cat. no. ab55878; Abcam), LC3 (1:1,000; cat. no. ab48394; Abcam), and $\beta$-actin (1:1,000; cat. no. ab3280; Abcam) at $20-27^{\circ} \mathrm{C}$ for $2 \mathrm{~h}$ before incubation with secondary antibodies conjugated to horseradish peroxidase-conjugated goat anti-rabbit Immunoglobulin G (1:5,000, cat. no. ab7074; Abcam) at $37^{\circ} \mathrm{C}$ for $20 \mathrm{~min}$ and visualized with enhanced chemiluminescence reagent (Vazyme). Immunoreactive bands were analyzed using Image Pro Plus 6.0 software (Media Cybernetics, Inc.).

Statistical analysis. Plots were constructed using GraphPad Prism 7 (GraphPad Software, Inc.), and the statistical analysis was performed using SPSS 19.0 (IBM Corp.) software. Normally distributed data are presented as the mean \pm SD. The non-normally distributed data are presented as the mean \pm interquartile range or as the median. One-way and two-way ANOVA were used for comparisons between groups, and the Bonferroni test was used for multiple comparisons. $\mathrm{P}<0.05$ was considered to indicate a statistically significant difference. All experiments were repeated three times.

\section{Results}

Effect of LPS on the morphology of HK-2 cells. The morphology of HK-2 cells changed following LPS treatment. Under the microscope, the HK-2 cells in the control group exhibited good adherence, clear outlines, tight cell-cell junctions and good cell growth (Fig. 1A). After the addition of LPS, in a time-dependent manner, HK-2 cells were rounded 
A
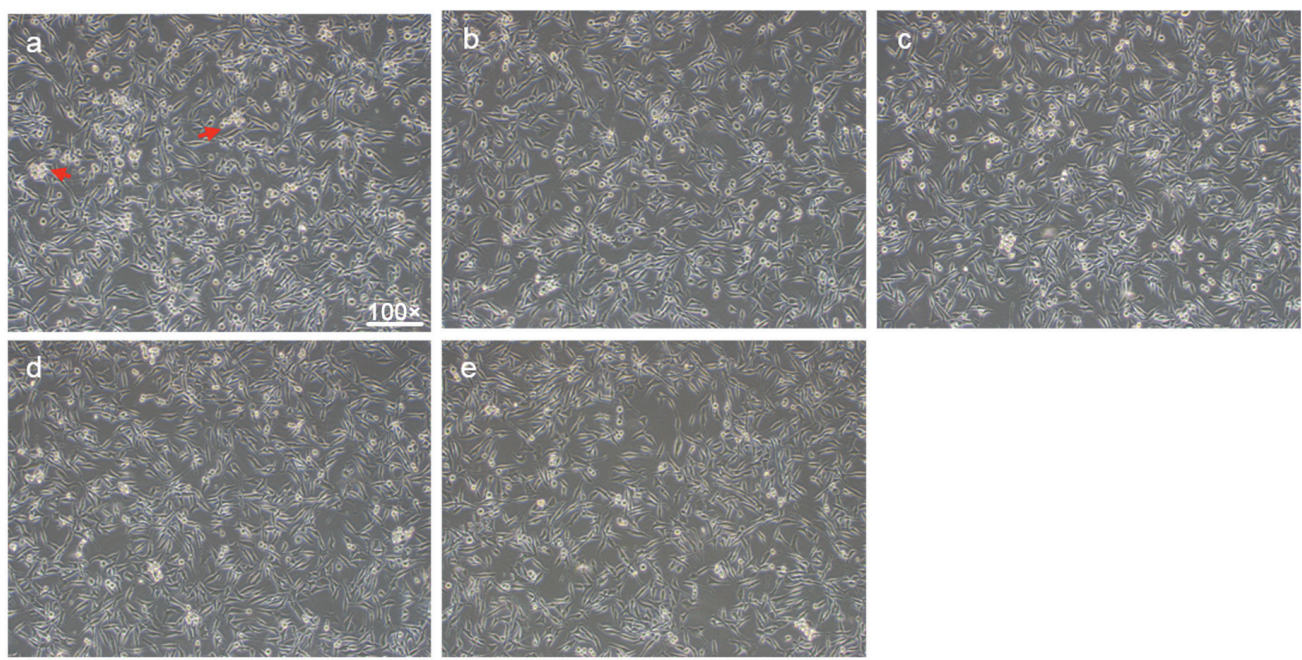

B

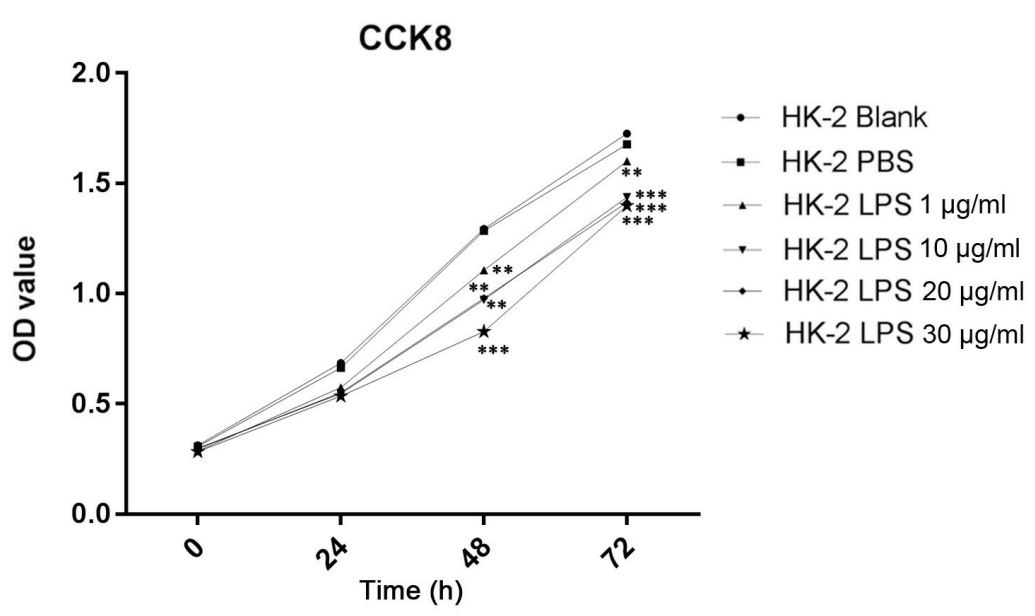

Figure 1. Effect of LPS on the morphology and viability of HK-2 cells. (A) Effect of LPS on the morphology of HK-2 cells after 24 h. (A-a) Control group. (A-b) LPS was $1 \mu \mathrm{g} / \mathrm{ml}$. (A-c) LPS $10 \mu \mathrm{g} / \mathrm{ml}$. (A-d) LPS $20 \mu \mathrm{g} / \mathrm{ml}$. (A-e) LPS $30 \mu \mathrm{g} / \mathrm{ml}$. (B) Effect of LPS on the viability of HK-2 cells. LPS, lipopolysaccharide. OD, optical density. ${ }^{* *} \mathrm{P}<0.01,{ }^{* * * *} \mathrm{P}<0.001$, HK-2 LPS $1 \mu \mathrm{g} / \mathrm{ml}$ vs. HK-2 LPS $10,20,30 \mu \mathrm{g} / \mathrm{ml}$.

into a slender or fusiform shape with poorly defined outlines, weaker cell-cell junctions, reduced adherence with reduced of HK-2 cell numbers (Fig. 1A). This finding suggested that LPS inhibited the growth of HK-2 cells.

Effects of LPS on the viability of HK-2 cells. After treatment of HK-2 cells with increasing concentrations of LPS up to $30 \mu \mathrm{g} / \mathrm{ml}$, CCK-8 assay results indicated that HK-2 cell viability decreased in response to LPS in a dose-dependent manner after $72 \mathrm{~h}(\mathrm{P}<0.01, \mathrm{P}<0.001$; Fig. 1B).

Effect of LPS on the expression of ATM in HK-2 cells. Following LPS stimulation, ATM mRNA expression increased in HK-2 cells, with the magnitude peaking when the dose of $10 \mu \mathrm{g} / \mathrm{ml}$ LPS was used. Increasing the dose of LPS to 20 and $30 \mu \mathrm{g} / \mathrm{ml}$ produced no further increases in ATM expression (Fig. 2).

Effect of LPS on the expression of inflammatory factors in $H K-2$ cells. Following $24 \mathrm{~h}$ of LPS treatment, the IL-6 and IL-1 $\beta$ mRNA expression levels increased in a dose-dependent manner compared with the control group (Fig. 3A and B).

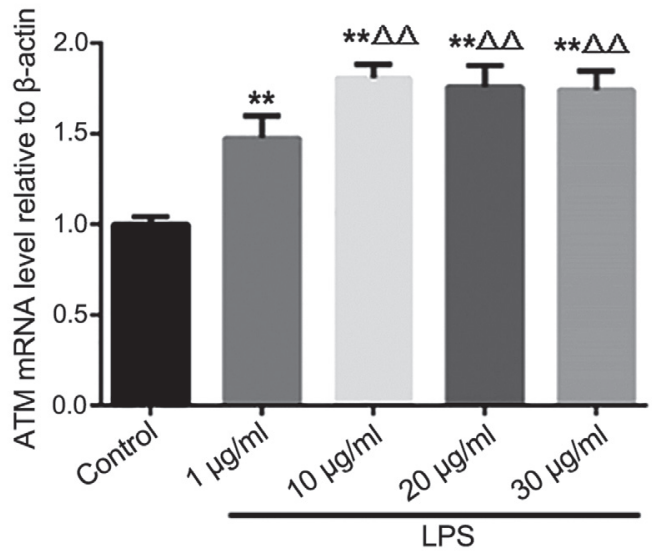

Figure 2. Effects of increasing concentrations of LPS on the expression of ATM mRNA in HK-2 cells. ${ }^{* *} \mathrm{P}<0.01$ vs. control and ${ }^{\Delta \Delta} \mathrm{P}<0.01$ vs. $1 \mu \mathrm{g} / \mathrm{ml}$ LPS. ATM, ataxia telangiectasia mutated; LPS, lipopolysaccharide.

The expression level of TNF- $\alpha$ mRNA was higher after 1 and $10 \mu \mathrm{g} / \mathrm{ml}$ LPS stimulation compared with the control group; 


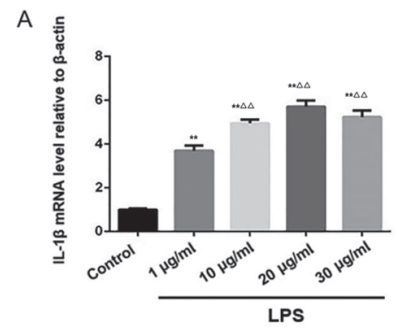

D

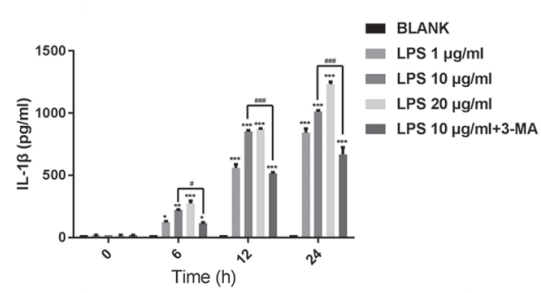

B

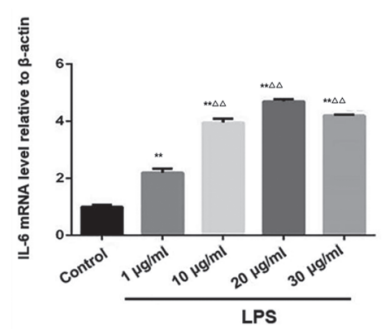

$\mathrm{E}$

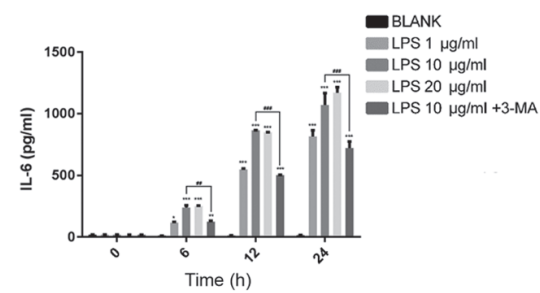

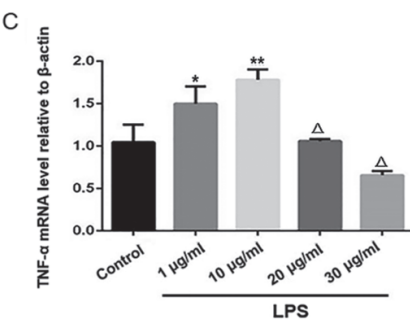

$\mathrm{F}$

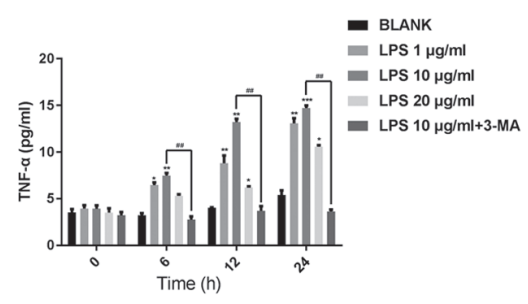

Figure 3. Effects of different concentrations of LPS on the expression of (A) IL-1 $\beta$, (B) IL-6, (C) TNF- $\alpha$ mRNA in HK-2 cells as detected using reverse transcription-quantitative PCR. " $\mathrm{P}<0.05$ and ${ }^{* *} \mathrm{P}<0.01$ vs. control and ${ }^{\Delta} \mathrm{P}<0.05$ and ${ }^{\Delta} \mathrm{P}<0.01 \mathrm{vs} .1 \mu \mathrm{g} / \mathrm{ml}$ LPS. Effects of different concentrations of LPS and concomitant 3-MA treatment on the concentrations of (D) IL-1 $\beta$, (E) IL-6 and (F) TNF- $\alpha$ in HK-2 cells as measured using ELISA. "P $<0.05$, ** P $<0.01$ and ${ }^{* * * *} \mathrm{P}<0.001$, vs. BLANK, ${ }^{\# \#} \mathrm{P}<0.01$ and ${ }^{\# \# \#} \mathrm{P}<0.001$, vs. $10 \mu \mathrm{g} / \mathrm{ml}$ LPS. LPS, lipopolysaccharide; IL, interleukin; TNF- $\alpha$, tumor necrosis factor- $\alpha$.

however, the expression level of TNF- $\alpha$ following treatment with 20 and $30 \mu \mathrm{g} / \mathrm{ml}$ LPS was lower compared with treatment with $1 \mu \mathrm{g} / \mathrm{ml}$ LPS (Fig. 3C). The results of RT-qPCR analysis were subsequently verified using ELISA. LPS treatment increased IL- $1 \beta$, IL- 6 and TNF- $\alpha$ expression in a dose-dependent manner. The levels of the three inflammatory factors aforementioned peaked when a dose of $10 \mu \mathrm{g} / \mathrm{ml}$ LPS used, and were reduced with the concomitant treatment with the autophagy inhibitor 3-MA (Fig. 3D-F). These results suggested that LPS induced an inflammatory response in HK-2 cells. Based on the findings of this experiment, LPS at a concentration of $10 \mu \mathrm{g} / \mathrm{ml}$ was selected for subsequent experiments.

Effect of ATM lentiviral interference vector on ATM-2 expression in $\mathrm{HK}-2$ cells. Following the construction of lentivirus-mediated ATM-knockdown system and using it to transfect HK-2 cells, the linearization of the interference carrier and the carrier map are shown in Fig. 4A, and the enzyme digestion map is shown in Fig. 4B. Lentiviral vectors encoding shRNA2143 and shRNA2144 were more efficient in knocking down ATM expression and compared with shRNA2142 and shRNA2145 (Fig. 4C). To validate this observation, HK-2 cells were transfected with lentiviruses encoding shRNA2143 and shRNA2144, followed by western blot analysis. ATM protein expression was significantly reduced following transfection with shRNA2143 and shRNA2144 (P<0.05; Fig. 4D and E).

Effect of ATM knockdown on the levels of inflammatory cytokines in HK-2 cells. After transfection of HK-2 cells with shRNA2143- and shRNA2144-encoding lentiviral particles, no statistically significant differences between the shRNA2143 and shRNA2144 interference groups were observed in the levels of IL-1 $\beta$, IL- 6 and TNF- $\alpha$ in the absence of LPS. Following LPS stimulation and transfection with shRNA2143 or shRNA2144, the levels of IL-1 $\beta$, IL- 6 and TNF- $\alpha$ were significantly lower compared with the NC shRNA group (Fig. 5A-C). The result suggested that downregulation of ATM expression can partially reduce the expression of inflammatory cytokines caused by LPS.

Effect of downregulating ATM expression on in HK-2 cell autophagy. After HK-2 cells were transfected with shRNA2143 and shRNA2144, of HK-2 cell autophagy was detected using immunofluorescence and western blotting following LPS stimulation.

After LPS stimulation of HK-2 cells for $24 \mathrm{~h}$, immunofluorescence assay showed that the levels of autophagy after transfection with shRNA2143 and shRNA2144 were reduced compared with the control group (Fig. 6A). The result suggested that downregulation of ATM expression can reduce autophagy caused by LPS.

The protein expression levels of beclin-1 in HK-2 cells transfected with shRNA2143 and shRNA2144 were significantly lower compared with the NC shRNA group (Fig. 6B and C). Although the LC3I/II ratio exhibited a certain degree of reduction in LPS-treated shRNA2143 and shRNA2144 groups compared with NC shRNA, no statistically significant differences were observed (Fig. 6D). These observations suggested that downregulation of ATM expression can significantly reduce the beclin-1 expression. ATM may be involved in the autophagy of HK-2 cells induced by LPS.

\section{Discussion}

In the present study, a model of septic AKI was established using LPS-stimulated HK-2 cells. Treatment with LPS increased the expression of inflammatory factors in HK-2 cells. In HK-2 cells with ATM expression knocked down, the levels of autophagy and expression levels of inflammatory cytokines were reduced. Therefore, it may be hypothesized that ATM can increase the expression of inflammatory cytokines further by promoting autophagy, resulting in HK-2 cell damage. 
A

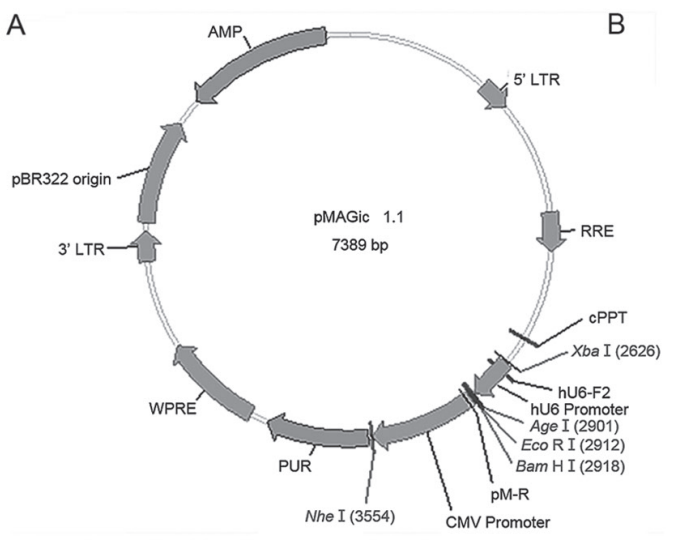

$B$

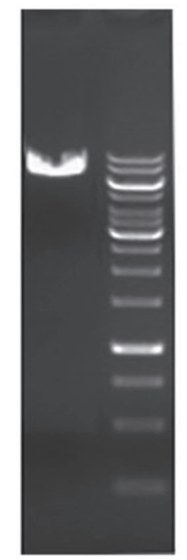

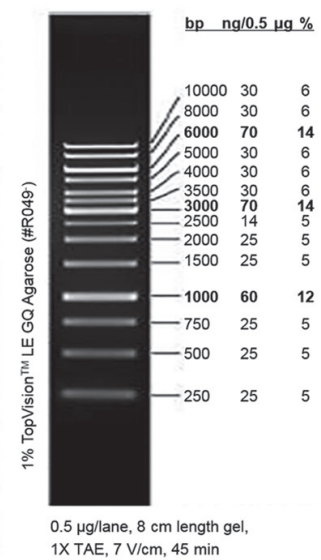

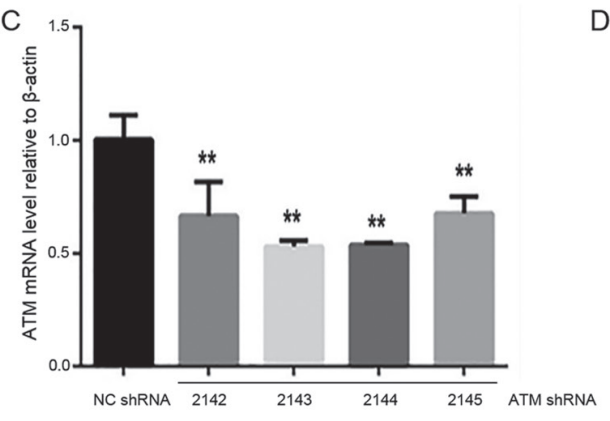

D

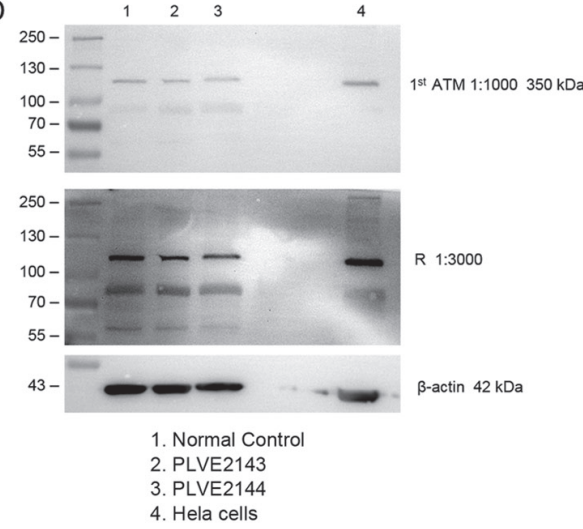

E

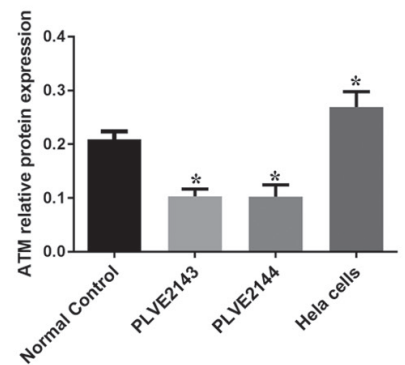

Figure 4. Construction of the ATM shRNA lentiviral vector and subsequent transfection. (A) Map of the lentiviral vector backbone used for cloning ATM shRNA-sequences. (B) Enzymatic digestion of the lentiviral vector to recover a $7.3 \mathrm{~kb}$ vector fragment. A $1 \mathrm{~kb}$ DNA ladder is provided next to the DNA gel as reference. (C) Effect of transfection with ATM lentiviral interference vectors encoding four different custom-designed ATM shRNAs on the expression of ATM mRNA in HK-2 cells. ${ }^{* *} \mathrm{P}<0.01$ vs. NC shRNA. (D) Western blot analysis of ATM protein expression following transfection with lentiviral vectors encoding shRNA2143 and shRNA2144 targeting ATM. (E) Western blot analysis of ATM protein expression. ${ }^{*}$ P $<0.05$ vs. NC shRNA. shRNA, short hairpin RNA; ATM, ataxia telangiectasia mutated; 1st ATM, primary antibody; R, repeat.

AKI is a severe clinical condition with high rates of morbidity and mortality due to a lack of effective treatment (26). The mortality rate of patients with septic AKI is $>70 \%$, significantly higher compared with patients with sepsis but without AKI (27). Conventionally the main pathophysiological mechanism of septic AKI was considered to be renal ischemia-induced hypoperfusion resulting from renal tubular perivascular dysfunction and acute tubular necrosis (ATN). However, renal pathology analysis in patients who succumbed to septic AKI revealed that $70 \%$ of those patients exhibited no ATN; instead, RTEC apoptosis was more common. In the case of constant or even increased renal blood flow, RTEC still undergo apoptosis, suggesting that renal hemodynamic changes are only part of the cause of septic AKI (28). Inflammation serves a key role in the pathophysiological mechanism of septic AKI and septic AKI is directly associated with the inflammatory response (29). However, the mechanism of this cycle remains unclear due to the complexity of the mechanism involved.

Bacterial endotoxin and inflammatory cytokines have been reported to be direct and important causes of renal injury (30). Endotoxins, of which LPS is an example, can stimulate severe inflammatory reactions in the body, resulting in the production of a large number of inflammatory factors, including TNF- $\alpha$, IL-1 $\beta$ and IL-6, in turn leading to oxidative stress, mitochondrial damage and energy depletion and finally RTEC apoptosis (31). LPS induces AKI via the induction of tubular epithelial cell apoptosis (32). It is crucial to explore therapeutic strategies to inhibit LPS-induced RTEC apoptosis in treating AKI. In the present study, LPS was used to stimulate HK-2 cells to establish a septic AKI model. After LPS stimulation, the morphology of HK-2 cells changed. Untreated cells exhibited good adherence, clear outlines and tight cell-cell junctions. By contrast, cells treated with LPS changed from round to shuttle shapes, the outline of the cells becoming blurred with the cell- cell junctions appearing loose. LPS-treated cells also appear low confluency with the number of cells reduced. The viability of HK-2 cells after LPS treatment was measured using CCK-8 assay, and it was found that LPS reduced cell viability in a dose-dependent manner. In addition, changes in the expression of inflammatory factors TNF- $\alpha$, IL-1 $\beta$ and IL- 6 were examined in this model. It was found that LPS increased the expression of TNF- $\alpha$, IL-1 $\beta$ and IL- 6 mRNA, with a dose of $10 \mu \mathrm{g} / \mathrm{ml}$ having the most potent effect, consistent with previous reports (33-35). These results suggest that the LPS-induced HK-2 cell injury model was successful, providing basis for further research.

Autophagy is a general term for the process by which intracellular materials are degraded by lysosomes under the regulation of proteins associated with autophagy. It is the basic catabolic mechanism that contributes to the routine recycling of cell mate- 

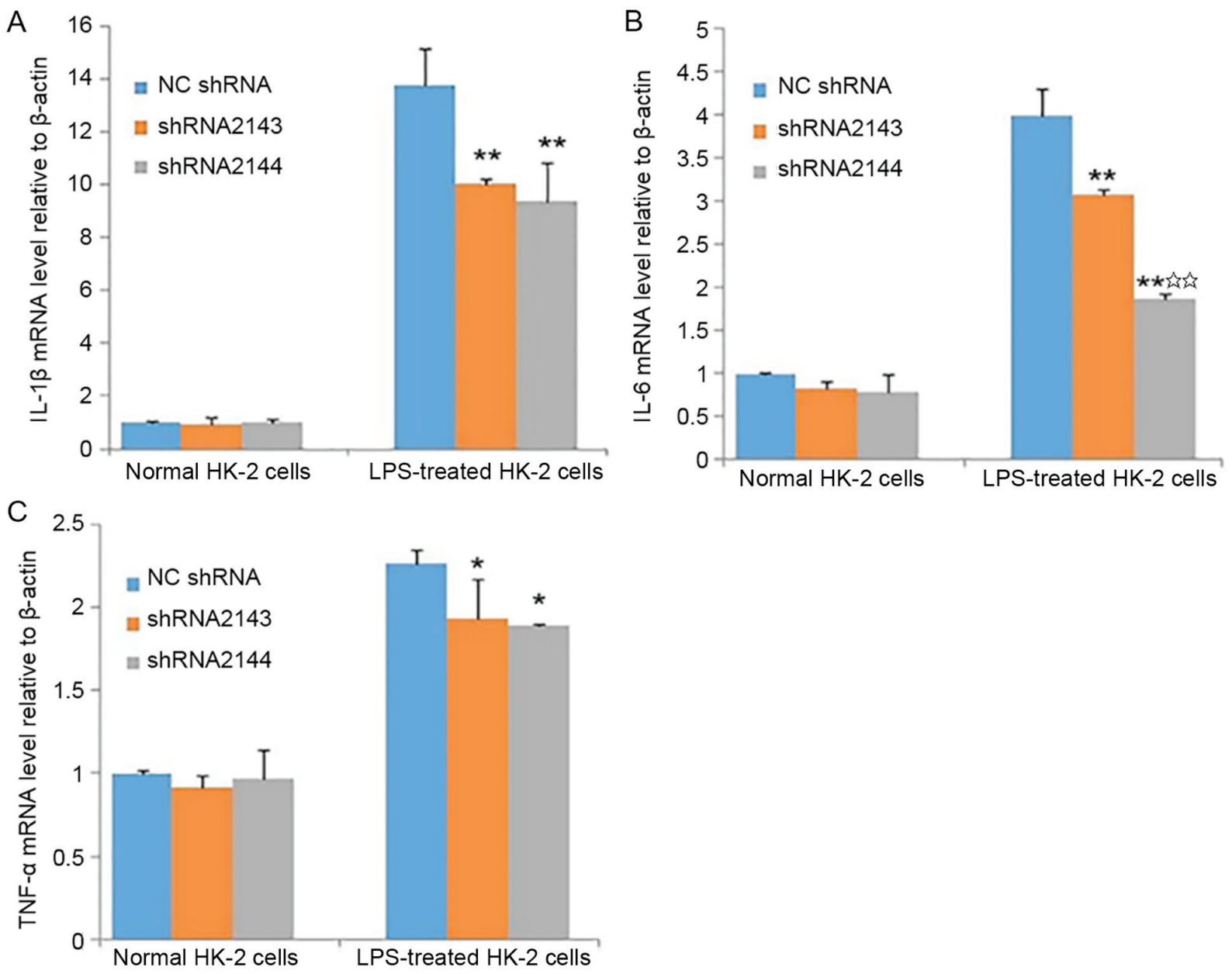

Figure 5. Effect of ATM knockdown on the expression of inflammatory cytokines in LPS-treated and untreated HK-2 cells. (A) Relative IL-1 $\beta$, (B) IL-6 and (C) TNF- $\alpha$ mRNA expression levels. " $\mathrm{P}<0.05$ and ${ }^{* *} \mathrm{P}<0.01$ vs. the NC shRNA group; ${ }^{*}{ }^{2} \mathrm{P}<0.01$ vs. shRNA2143. LPS, lipopolysaccharide; IL, interleukin; TNF- $\alpha$, tumor necrosis factor- $\alpha$; ATM, ataxia telangiectasia mutated; shRNA, short hairpin RNA.

rials by the turnover of dysfunctional cellular components (36). This process exists in physiological and pathophysiological processes of a number of different diseases (37). Indeed, autophagy occurs in most tissues under both physiological and pathological conditions $(16,38,39)$, which can be dramatically upregulated by unfavorable stimulus, including hypoxia and nutrient depletion (40). A previous study has demonstrated that excessive autophagy may lead to programmed cell death (41). However, autophagy is invariably linked with disease regardless of whether it is excessively inhibited or activated, since it serves a dual role in cell survival and death (42). In particular, similar studies have found that autophagy serves an important role in the pathogenesis of sepsis $(43,44)$.

The pathogenesis of LPS-induced AKI is closely associated with excessive inflammation (45). Pro-inflammatory cytokines are the major mediators of AKI induced by sepsis (46). Controlling the production of pro-inflammatory factors and downstream pro-inflammatory mediators may be an effective approach in AKI therapy (47). Kong et al (48) demonstrated that antithrombin III can ameliorate serum amyloid $\mathrm{P}$ component-induced renal damage by inhibiting inflammation, oxidative stress and apoptosis. In addition, Lu et al (49) came to the same conclusion in the medium contrast-induced AKI. In the present study, the role of ATM in LPS-induced septic AKI and its mechanism were explored. In a previous study, persistent inflammatory response causes DNA damage in RTEC, further activating the protein kinase ATM $(21,22)$. In a model of ischemia-reperfusion kidney injury, AMPK was found to activate the ATM-AMPK-TSC2-mTOR pathway, causing autophagy (23). In the present study, it was found that LPS stimulated HK-2 cells and caused an increase in intracellular ATM mRNA levels. To explore the role of ATM in LPS-induced HK-2 cell injury, an ATM-interfering lentivirus was constructed, which was used to transfect HK-2 cells to downregulate endogenous ATM expression. Following ATM knockdown, LPS-induced increase in the expression of inflammatory cytokines IL-6, IL-1 $\beta$ and TNF- $\alpha$ was partially reversed. Likewise, beclin-1 expression was significantly reduced compared with NC shRNA group, but no significant changes were observed in the LC3 II/I ratio. The role of autophagy in AKI has not been previously determined. At present, some studies have supported the notion that autophagy can protect RTEC cells and alleviate AKI deterioration $(17,18,50)$, whilst other studies suggest that autophagy will increase cell death $(37,51)$. It remains unclear whether autophagy protects RTEC against AKI and any associated mechanism. A previous study using a mouse model of sepsis induced by intraperitoneal LPS injection reported that the expression levels of LC3, beclin-1 and other genes associated with autophagy 
A
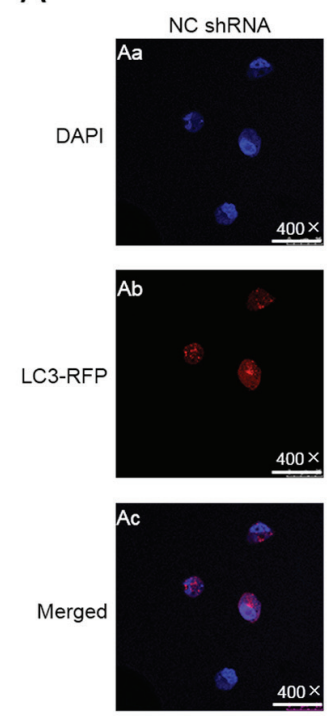

C

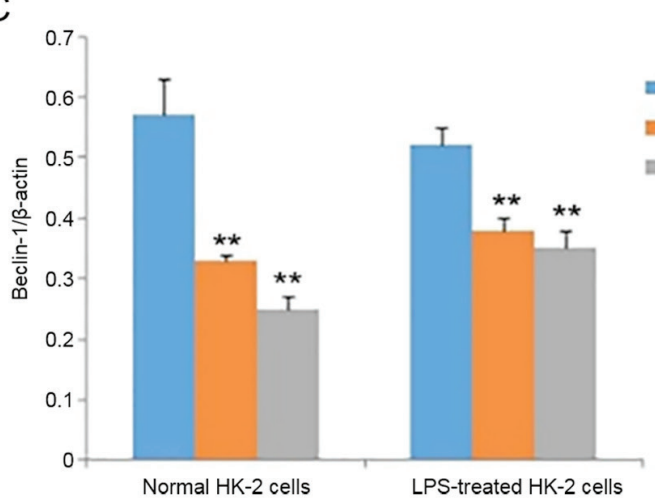

ShRNA2143
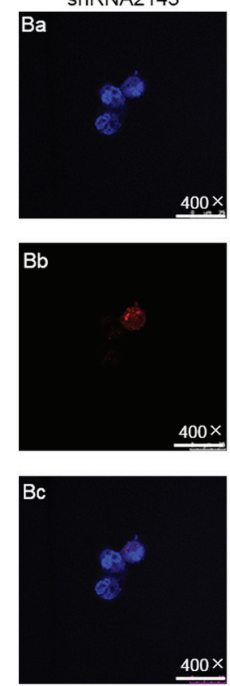

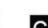

aNC ShRNA

an shRNA2143 플 ShRNA2144
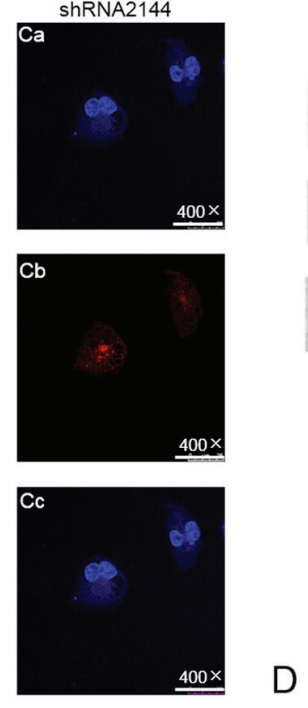

B

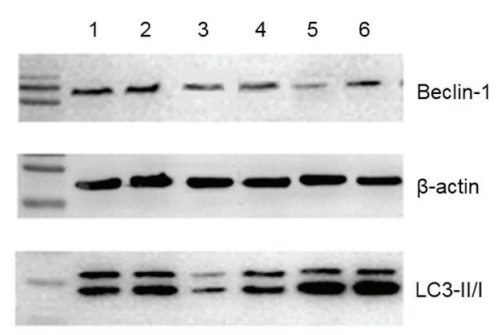

1. NC shRNA of Normal HK-2 cells

2. NC shRNA of LPS-treated HK-2 cells

3. shRNA2143 of Normal HK-2 cells

4. shRNA2143 of LPS-treated HK-2 cells

5. shRNA2144 of Normal HK-2 cells

6. shRNA2144 of LPS-treated HK-2 cells

Figure 6. Effect of ATM on HK-2 cell autophagy. (A) Immunofluorescence to assess the effect of ATM expression on autophagy in HK-2 cells. Blue represents DAPI staining and red represents LC3-RFP staining. (B) Western blot analysis of beclin-1 and LC3 II/I expression in LPS-treated HK-2 cells transfected with shRNA targeting ATM. Quantified densitometry data for (C) beclin-1 expression and (D) LC3 II/I ratio. ${ }^{* *} \mathrm{P}<0.01$ vs. NC shRNA. RFP, red fluorescent protein; shRNA, short hairpin RNA; LPS, lipopolysaccharide; ATM, ataxia telangiectasia mutated.

were elevated, and the secretion of inflammatory factors IL-1 $\beta$ and IL-18 was also significantly increased (52). It has also been reported that reducing autophagy promotes inflammatory responses and subsequent cell death (53). Autophagy has been shown to be upregulated in numerous AKI models (54). In the present study, it was found that autophagy aggravated the inflammatory response and cellular damage in HK-2 cells treated with LPS for $24 \mathrm{~h}$. When shRNA2143 and shRNA2144 were used to knockdown ATM expression, it was found that the levels of autophagy and inflammatory factors were also significantly reduced following $24 \mathrm{~h}$ LPS treatment. This suggests that the level of autophagy is reduced when ATM is downregulated. Therefore, it can be hypothesized that LPS may induce autophagy in HK-2 cells through the ATM pathway, resulting in the upregulation of pro-inflammatory cytokines. To the best of our knowledge, this was the first time that ATM was found to increase the expression of inflammatory cytokines by promoting autophagy, resulting in HK-2 cell damage.

In conclusion, the present study revealed that LPS can reduce HK-2 cell viability, and increase autophagy and the expression of inflammatory cytokines IL-1 $\beta$ and IL-6, ATM. By contrast, downregulation of ATM levels in HK-2 cells can reduce the levels of inflammatory cytokines and autophagy in
LPS-induced HK-2 cells. Autophagy can exacerbate inflammatory responses and cellular damage, however, the mechanism by which ATM affects LPS-induced inflammatory response and autophagy in AKI renal tubular epithelial cells remains unclear. The present study provides a new direction and lays a foundation for the future treatment of AKI.

\section{Acknowledgements}

Not applicable.

\section{Funding}

This work was supported by grants from the Wenzhou Committee of Science and Technology of China (grant nos. Y20170055, ZS2017008 and Y20180159) and the Zhejiang Province Natural Science Foundation (grant nos. LQ19H050002 and LY15H050008).

\section{Availability of data and materials}

The datasets used and/or analyzed during the current study are available from the corresponding author on reasonable request. 


\section{Authors' contributions}

CFZ and YZ wrote the first draft of manuscript. CFZ, YYH and $\mathrm{BCC}$ contributed to the conception and design of the research. MMW, YX, XC and MS contributed to the experiments and analysis of the data. YZ, YL, CSC and JGP contributed to the analysis and interpretation of the data. All authors critically revised the manuscript, and agreed to be fully accountable for ensuring the integrity and accuracy of the work, and read and approved the final manuscript.

\section{Ethics approval and consent to participate}

Not applicable.

\section{Patient consent for publication}

Not applicable.

\section{Competing interests}

The authors declare that they have no competing interests.

\section{References}

1. Bagshaw SM, Uchino S, Bellomo R, Morimatsu H, Morgera S, Schetz M, Tan I, Bouman C, Macedo E, Gibney N, et al: Septic acute kidney injury in critically ill patients: Clinical characteristics and outcomes. Clin J Am Soc Nephrol 2: 431-439, 2007.

2. Singer M, Deutschman CS, Seymour CW, Shankar-Hari M, Annane D, Bauer M, Bellomo R, Bernard GR, Chiche JD, Coopersmith CM, et al: The third international consensus definitions for sepsis and septic shock (Sepsis-3). JAMA 315: 801-810, 2016.

3. Holthoff JH, Wang Z, Patil NK, Gokden N and Mayeux PR: Rolipram improves renal perfusion and function during sepsis in the mouse. J Pharmacol Exp Ther 347: 357-364, 2013.

4. Howell GM, Gomez H, Collage RD, Loughran P, Zhang X, Escobar DA, Billiar TR, Zuckerbraun BS and Rosengart MR: Augmenting autophagy to treat acute kidney injury during endotoxemia in mice. PLoS One 8: e69520, 2013.

5. Bagshaw SM, George C and Bellomo R; ANZICS Database Management Committee: Early acute kidney injury and sepsis: A multicentre evaluation. Crit Care 12: R47, 2008.

6. Wang F, Zhang G, Lu Z, Geurts AM, Usa K, Jacob HJ, Cowley AW, Wang N and Liang M: Antithrombin III/SerpinC1 insufficiency exacerbates renal ischemia/reperfusion injury. Kidney Int 88: 796-803, 2015.

7. Chen LW, Chen W, Hu ZQ, Bian JL, Ying L, Hong GL, Qiu QM, Zhao GJ and Lu ZQ: Protective effects of growth arrest-specific protein 6 (Gas6) on sepsis-induced acute kidney injury. Inflammation 39: 575-582, 2016.

8. Yohannes $\mathrm{S}$ and Chawla LS: Evolving practices in the management of acute kidney injury in the ICU (Intensive Care Unit). Clin Nephrol 71: 602-607, 2009.

9. Gómez H, Kellum JA and Ronco C: Metabolic reprogramming and tolerance during sepsis-induced AKI. Nat Rev Nephrol 13: 143-151, 2017.

10. Luo CJ, Luo F, Zhang L, Xu Y, Cai GY, Fu B, Feng Z, Sun XF and Chen XM: Knockout of interleukin-17A protects against sepsis-associated acute kidney injury. Ann Intensive Care 6: 56, 2016.

11. Chunzhi G, Zunfeng L, Chengwei Q, Xiangmei B and Jingui Y: Hyperin protects against LPS-induced acute kidney injury by inhibiting TLR4 and NLRP3 signaling pathways. Oncotarget 7: $82602-82608,2016$

12. Xu C, Chang A, Hack BK, Eadon MT, Alper SL and Cunningham PN: TNF-mediated damage to glomerular endothelium is an important determinant of acute kidney injury in sepsis. Kidney Int 85: 72-81, 2014.
13. Ahn JM, You SM, Lee YM, Oh SW, Ahn SY, Kim S, Chin HJ, Chae DW and Na KY: Hypoxia-inducible factor activation protects the kidney from gentamicin-induced acute injury. PLoS One 7: e48952, 2012.

14. Sutton TA, Hato T, Mai E, Yoshimoto M, Kuehl S, Anderson M, Mang H, Plotkin Z, Chan RJ and Dagher PC: p53 Is renoprotective after ischemic kidney injury by reducing inflammation. J Am Soc Nephrol 24: 113-124, 2013.

15. Klionsky DJ and Emr SD: Autophagy as a regulated pathway of cellular degradation. Science 290: 1717-1721, 2000.

16. Levine B and Kroemer G: Autophagy in the pathogenesis of disease. Cell 132: 27-42, 2008.

17. Leventhal JS, Ni J, Osmond M, Lee K, Gusella GL, Salem F and Ross MJ: Autophagy limits endotoxemic acute kidney injury and alters renal tubular epithelial cell cytokine expression. PLoS One 11: e0150001, 2016.

18. Mei S, Livingston M, Hao J, Li L, Mei C and Dong Z: Autophagy is activated to protect against endotoxic acute kidney injury. Sci Rep 6: 22171, 2016.

19. Yu JH, Cho SO, Lim JW, Kim N and Kim H: Ataxia telangiectasia mutated inhibits oxidative stress-induced apoptosis by regulating heme oxygenase-1 expression. Int J Biochem Cell Biol 60: 147-156, 2015.

20. Moser BA, Subramanian L, Khair L, Chang YT and Nakamura TM: Fission yeast Tel1 (ATM) and Rad3 (ATR) promote telomere protection and telomerase recruitment. PLoS Genet 5: e1000622, 2009.

21. Abraham RT: Cell cycle checkpoint signaling through the ATM and ATR kinases. Genes Dev 15: 2177-2196, 2001.

22. Bencokova Z, Kaufmann MR, Pires IM, Lecane PS, Giaccia AJ and Hammond EM: ATM activation and signaling under hypoxic conditions. Mol Cell Biol 29: 526-537, 2009.

23. Wang LT, Chen BL, Wu CT, Huang KH, Chiang CK and Hwa Liu S: Protective role of AMP-activated protein kinase-evoked autophagy on an in vitro model of ischemia/reperfusion-induced renal tubular cell injury. PLoS One 8: e79814, 2013.

24. Livak KJ and Schmittgen TD: Analysis of relative gene expression data using real-time quantitative PCR and the 2 (-Delta Delta C (T)) method. Methods 25: 402-408, 2001.

25. Jung G, Roh J, Lee H, Gil M, Yoon DH, Suh C, Jang S, Park CJ, Huh J and Park CS: Autophagic markers BECLIN 1 and LC3 are associated with prognosis of multiple myeloma. Acta Haematol 134: 17-24, 2015.

26. Dellepiane S, Marengo M and Cantaluppi V: Detrimental cross-talk between sepsis and acute kidney injury: New pathogenic mechanisms, early biomarkers and targeted therapies. Crit Care 20: 61, 2016.

27. Jackson WL Jr: Acute renal failure and sepsis. N Engl J Med 351: 2347-2349, 2004

28. Langenberg C, Wan L, Egi M, May CN and Bellomo R: Renal blood flow in experimental septic acute renal failure. Kidney Int 69: 1996-2002, 2006.

29. Fani F, Regolisti G, Delsante M, Cantaluppi V, Castellano G, Gesualdo L, Villa G and Fiaccadori E: Recent advances in the pathogenetic mechanisms of sepsis-associated acute kidney injury. J Nephrol 31: 351-359, 2018.

30. Chen L, Yang S, Zumbrun EE, Guan H, Nagarkatti PS and Nagarkatti M: Resveratrol attenuates lipopolysaccharide-induced acute kidney injury by suppressing inflammation driven by macrophages. Mol Nutr Food Res 59: 853-864, 2015.

31. Deng SY, Zhang LM, Ai YH, Pan PH, Zhao SP, Su XL, Wu DD, Tan HY, Zhang LN and Tsung A: Role of interferon regulatory factor-1 in lipopolysaccharide-induced mitochondrial damage and oxidative stress responses in macrophages. Int J Mol Med 40: 1261-1269, 2017

32. Li T, Zhao J, Miao S, Xu Y, Xiao X and Liu Y: Dynamic expression and roles of sequestome-1/p62 in LPS-induced acute kidney injury in mice. Mol Med Rep 17: 7618-7626, 2018.

33. Jiao XY, Shen YQ and Li KS: The correlation between cytokine production by cerebral cortical glial cells and brain lateralization in mice. Neuromodulation 11: 23-32, 2008.

34. Johnson RL, Murray ST, Camacho DK and Wilson CG: Vagal nerve stimulation attenuates IL-6 and TNFa expression in respiratory regions of the developing rat brainstem. Respir Physiol Neurobiol 229: 1-4, 2016.

35. Lee WS, Shin JS, Jang DS and Lee KT: Cnidilide, an alkylphthalide isolated from the roots of Cnidium officinale, suppresses LPS-induced NO, PGE2, IL-1 $\beta$, IL- 6 and TNF- $\alpha$ production by AP-1 and NF- $\kappa$ B inactivation in RAW 264.7 macrophages. Int Immunopharmacol 40: 146-155, 2016. 
36. Kim KH and Lee MS: Autophagy as a crosstalk mediator of metabolic organs in regulation of energy metabolism. Rev Endoc Metab Disord 15: 11-20, 2014.

37. Shen HM and Codogno P: Autophagic cell death: Loch Ness monster or endangered species? Autophagy 7: 457-465, 2011

38. Shintani T and Klionsky DJ: Autophagy in health and disease: A double-edged sword. Science 306: 990-995, 2004.

39. Levine B and Yuan J: Autophagy in cell death: An innocent convict? J Clin Invest 115: 2679-2688, 2005.

40. Casado P, Bilanges B, Rajeeve V, Vanhaesebroeck B and Cutillas PR: Environmental stress affects the activity of metabolic and growth factor signaling networks and induces autophagy markers in MCF7 breast cancer cells. Mol Cell Proteomics 13 836-848, 2014

41. Shi R, Weng J, Zhao L, Li XM, Gao TM and Kong J: Excessive autophagy contributes to neuron death in cerebral ischemia. CNS Neurosci Ther 18: 250-260, 2012.

42. Livingston MJ and Dong Z: Autophagy in acute kidney injury. Semin Nephrol 34: 17-26, 2014.

43. Chien WS, Chen YH, Chiang PC, Hsiao HW, Chuang SM, Lue SI and Hsu C: Suppression of autophagy in rat liver at late stage of polymicrobial sepsis. Shock 35: 506-511, 2011.

44. Hsieh CH, Pai PY, Hsueh HW, Yuan SS and Hsieh YC: Complete induction of autophagy is essential for cardioprotection in sepsis Ann Surg 253: 1190-1200, 2011.

45. Şen V, Uluca Ü, Ece A, Güneş A, Zeytun H, Arslan S, Kaplan I, Türkçü G and Tekin R: Role of Ankaferd on bacterial translocation and inflammatory response in an experimental rat model of intestinal obstruction. Int J Clin Exp Med 7: 2677-2686, 2014.

46. Sang HS, Lee KE, Kim IJ, Kim O, Kim CS, Choi JS, Choi HI, Bae EH, Ma SK, Lee JU and Kim SW: Alpha-lipoic acid attenuates lipopolysaccharide-induced kidney injury. Clin Exp Nephrol 19: 82-91, 2015.
47. Xiang H, Hu B, Li Z and Li J: Dexmedetomidine controls systemic cytokine levels through the cholinergic anti-inflammatory pathway. Inflammation 37: 1763-1770, 2014.

48. Kong Y, Yin J, Cheng D, Lu Z, Wang N, Wang F and Liang M: Antithrombin III attenuates AKI following acute severe pancreatitis. Shock 49: 572-579, 2018.

49. Lu Z, Cheng D, Yin J, Wu R, Zhang G, Zhao Q, Wang N, Wang F and Liang M: Antithrombin III protects against contrast-induced nephropathy. EBioMedicine 17: 101-107, 2017.

50. Chen K, Dai H, Yuan J, Chen J, Lin L, Zhang W, Wang L, Zhang J, Li K and He Y: Optineurin-mediated mitophagy protects renal tubular epithelial cells against accelerated senescence in diabetic nephropathy. Cell Death Dis 9: 105, 2018.

51. Lu B, Capan E and Li C: Autophagy induction and autophagic cell death in effector T cells. Autophagy 3: 158-159, 2007.

52. Nakahira K, Haspel JA, Rathinam VA, Lee SJ, Lam HC, Rabinovitch M, et al: Autophagy proteins regulate innate immune response by inhibiting NALP3 inflammasome-mediated mitochondrial DAN release. In: American Thoracic Society 2011 International Conference, May 13-18, 2011 • Denver Colorado; 2011. pp. A1077-A1077.

53. Carchman EH, Rao J, Loughran PA, Rosengart MR and Zuckerbraun BS: Heme oxygenase-1-mediated autophagy protects against hepatocyte cell death and hepatic injury from infection/sepsis in mice. Hepatology 53: 2053-2062, 2011.

54. Kaushal GP: Autophagy protects proximal tubular cells from injury and apoptosis. Kidney Int 82: 1250-1253, 2012.

c) (i) $(-)$ This work is licensed under a Creative Commons Attribution-NonCommercial-NoDerivatives 4.0 International (CC BY-NC-ND 4.0) License. 